\title{
Cue-Mediated Recruitment in a Swarm-Founding Wasp: Successful Foragers Induce Nestmates to Search off Nest for a Scented Carbohydrate Resource
}

\author{
Teresa I. Schueller and Robert L. Jeanne \\ Departments of Entomology and Zoology, University of Wisconsin-Madison, 546 Russell Labs, 1630 Linden Drive, \\ Madison, WI 53706, USA \\ Correspondence should be addressed to Teresa I. Schueller, teresa.schueller@uwc.edu
}

Received 26 February 2012; Revised 4 May 2012; Accepted 6 May 2012

Academic Editor: James Charles Nieh

Copyright (C 2012 T. I. Schueller and R. L. Jeanne. This is an open access article distributed under the Creative Commons Attribution License, which permits unrestricted use, distribution, and reproduction in any medium, provided the original work is properly cited.

\begin{abstract}
The ability of social insect colonies to recruit nestmates to profitable resources increases colony-wide foraging efficiency by providing individuals with information that narrows their search for resources. Here we ask if for the Neotropical swarm-founding wasp Polybia occidentalis naïve nestmates are able to use food-scent cues from rich carbohydrate resources brought to the nest by successful foragers to orient to off nest resources. Foragers were allowed to freely visit a training dish containing a scented sucrose solution. At a second location, in a different direction from the nest, two sucrose-filled dishes were offered, one with the training scent and one with an alternate scent. Naïve foragers preferentially chose the training scent over the alternate scent, indicating that natural rates of resource inflow to the nest are sufficient to induce nestmates to forage at resources with a specific scent. Naïve foragers did not forage more often at the location at which the active foragers were foraging, an indication that directional information is not communicated in this species. The total number of foraging trips made by a colony's foragers was not determined by the size of the foraging force, but rather by the average individual foraging rate for the colony.
\end{abstract}

\section{Introduction}

Recruitment in social insects has been variously defined as communication that brings individuals to where work is needed [1], “(a)ny behavior that results in an increase in the number of individuals at a particular place" [2, page 115], and "the local increase of workers cooperating at a particular place" [3, page 29]. Recruitment to food enables foragers to exploit patchily distributed resources more efficiently than is possible by independent searching, because it allows them to make use of information that reduces the uncertainty of finding the resource $[4,5]$. For species that store excess food reserves in the nest, recruitment allows the colony to more quickly and fully exploit bonanzas that temporarily exceed immediate demand $[6,7]$.

Although recruitment is often narrowly defined as exclusively signal mediated [8-10], it may also be mediated by social cues $[2,3,6,7,11-14]$. Thus, one may distinguish between "signal-based recruitment" and "cue-based recruitment." The waggle dance of the honey bee and pheromone trails of ants are examples of the former, whereas the social wasps' use of food odors brought to the nest and local enhancement at feeding sites are examples of cue-based recruitment to food resources $[2,15]$. Although most ant and many bee species have evolved signals used in recruitment to food, they no doubt also make use of social cues [3, 1519]. In contrast, the social wasps appear to lack signal-based recruitment to food and to rely exclusively on cue-based mechanisms $[6,7,14,20-23]$ (with the exception of the hornet Vespa mandarinia, which uses a pheromone signal to recruit nestmates to assist in attacking honey bee colonies [24]).

Foraging and recruitment to food have been little studied in the over 230 species of Neotropical swarm-founding wasps in the polistine tribe Epiponini [14]. This group is known for its broad range of colony sizes, complex social structure, 
and striking ecological dominance $[25,26]$. Several studies of Polybia occidentalis provide insight into the foraging process in this group. $P$. occidentalis is characterized by moderately large colony size and by the ability to store nectar in the nest $[26,27]$. During the founding stage, foraging rates are directly correlated with the number of cells in the nest and with the number of larvae in later developmental stages, both of which are indications of colony demand [28].

The coordination of colony-level foraging in $P$. occidentalis occurs without the use of food-source scent-marking [29]. Instead, naïve foragers use cues encountered both in the nest and at the food site to help locate food. Direct introduction of a scented sugar solution into the nest causes an increase in the number of foragers departing from the nest [30]. At least some of these site-naïve individuals learn the food-associated scent and use it as a cue to help locate the source in the field [7]. In a study showing the importance of local enhancement as a cue, foragers bringing a rich, unscented sugar solution to the nest caused an increase in the number of new individuals arriving at a feeding station, where they overwhelmingly chose the food dish at which conspecifics were feeding over an identical dish without conspecifics [6].

In two later studies on this species [7, 30], done at the same field site as the present study, large amounts of scented sugar solution were added directly to the nest, rather than letting foragers bring the resource to the nest from a dish to which they were trained. This approach has two advantages: (1) the large influxes of food stimulated a foraging response that rose enough above background rates to be quantifiable and (2) the output response could be precisely quantified in terms of the known amount of resource input. These studies also demonstrated that the recruiting effects occur independently of the behavior of returning foragers in the nest.

However, these prior studies leave several questions unanswered. Schueller et al. [7] added $40 \mathrm{~mL}$ of a $2.0 \mathrm{M}$ sucrose solution to the nest, an amount equivalent to more than 6,000 crop loads (average forager crop size is approximately $6.6 \mu \mathrm{L}$ ) [31]. This translates to a foraging rate equivalent to over $100 \mathrm{loads} / \mathrm{min}$ during the 60 minutes of the study. As this rate is more than 35 times the maximum foraging rate documented for this species [32], it leaves open the question of whether the much smaller amounts of scented solution brought back by a few foragers can have the same inducing effect. Although Hrncir et al. [6] let trained foragers return to the nest from a distant dish, because they used unscented sugar solutions, no inferences could be made about the wasps' use of odor cues. Finally, none of these studies directly addressed the question of whether returning foragers provide information about the direction of the resource. In many swarm-founding wasp species, during the dispersal of a swarm from its parental nest, scout wasps lay scent spots on vegetation between the old nest and the new site they have chosen $[26,33,34]$. Although some Agelaia spp. have been shown not to use this system to recruit nestmates to a rich protein source [20], whether or not in $P$. occidentalis uses this type of recruitment system for either protein or carbohydrate resources has not been tested.
Here, we address three questions. Firstly, we ask whether the influx of a profitable, scented carbohydrate resource brought to the nest by active foragers is sufficient to induce inactive foragers to begin foraging and searching for a resource with the same scent. Secondly, we ask whether more foragers arrive at the resource being exploited by the active foragers than at an identical resource in a different direction, which could indicate that directional information is being conveyed at the nest. Finally, we ask whether the magnitude of the colony's response to the influx of high-quality food is correlated with colony demand, as measured by number of adults and/or brood, and how the colony-level response is parsed at the individual level.

\section{Methods}

The investigation was conducted on the private property of the Hagnauer family and the adjacent grounds of Hacienda La Pacifica, near Cañas, Guanacaste, Costa Rica (10.450N, 85.125W), during June and July 2010, shortly after the beginning of the rainy season. Historically, this area was dry forest. Now the Hagnauer property is predominantly pastureland with scattered trees, whereas the grounds of Hacienda La Pacifica have wooded patches with openings planted to lawns and ornamentals. P. occidentalis nests are abundant in such disturbed areas; the wasps often construct nests in trees and shrubs along fence lines or roadsides. Colonies were sought within a radius of approximately $1 \mathrm{~km}$ from the study site and then moved to locations convenient for conducting the experiment, typically into isolated trees in pastures or yards. We conducted thorough searches of potential nest sites in the area to be sure that no other $P$. occidentalis colonies were close enough for their foragers to interfere with our experiments. Because these wasps are unable to fly in the dark, nests were moved at night to ensure that all colony members were present in the nest during the relocation process. At their new locations, nests were attached to branches about a meter high using metal binder clips, wire, or plastic zip ties. The proximal ends of the branches were coated for approximately $2.5 \mathrm{~cm}$ with Tanglefoot (Tanglefoot Co., Grand Rapids, MI, USA) to prevent predation by ants.

Two feeding stations were used during this investigation. The training station was used to mark foragers and, after marking was completed, was used to supply a sucrose solution scented with the training scent to trained foragers. The testing station offered a choice of scented sucrose solutions, one with the training scent and another with an alternate scent. Both stations consisted of Plexiglas tabletops $(43 \mathrm{~cm} \times 30 \mathrm{~cm})$ attached to tripods and adjusted so that the dishes were approximately $0.75 \mathrm{~m}$ above the ground and clear of nearby vegetation. The training and testing stations were located $5 \mathrm{~m}$ apart and $5 \mathrm{~m}$ downwind from the colony (Figure 1). A 2.0 M sucrose solution, either unscented (during training and marking) or containing a $2 \%$ scent extract (during testing), was used in all dishes. The sucrose solution was placed in glass feeder dishes $(6.5 \mathrm{~cm}$ diameter, $1.75 \mathrm{~cm}$ deep)_one placed in the center of the training station, and two placed approximately $11 \mathrm{~cm}$ apart at the 


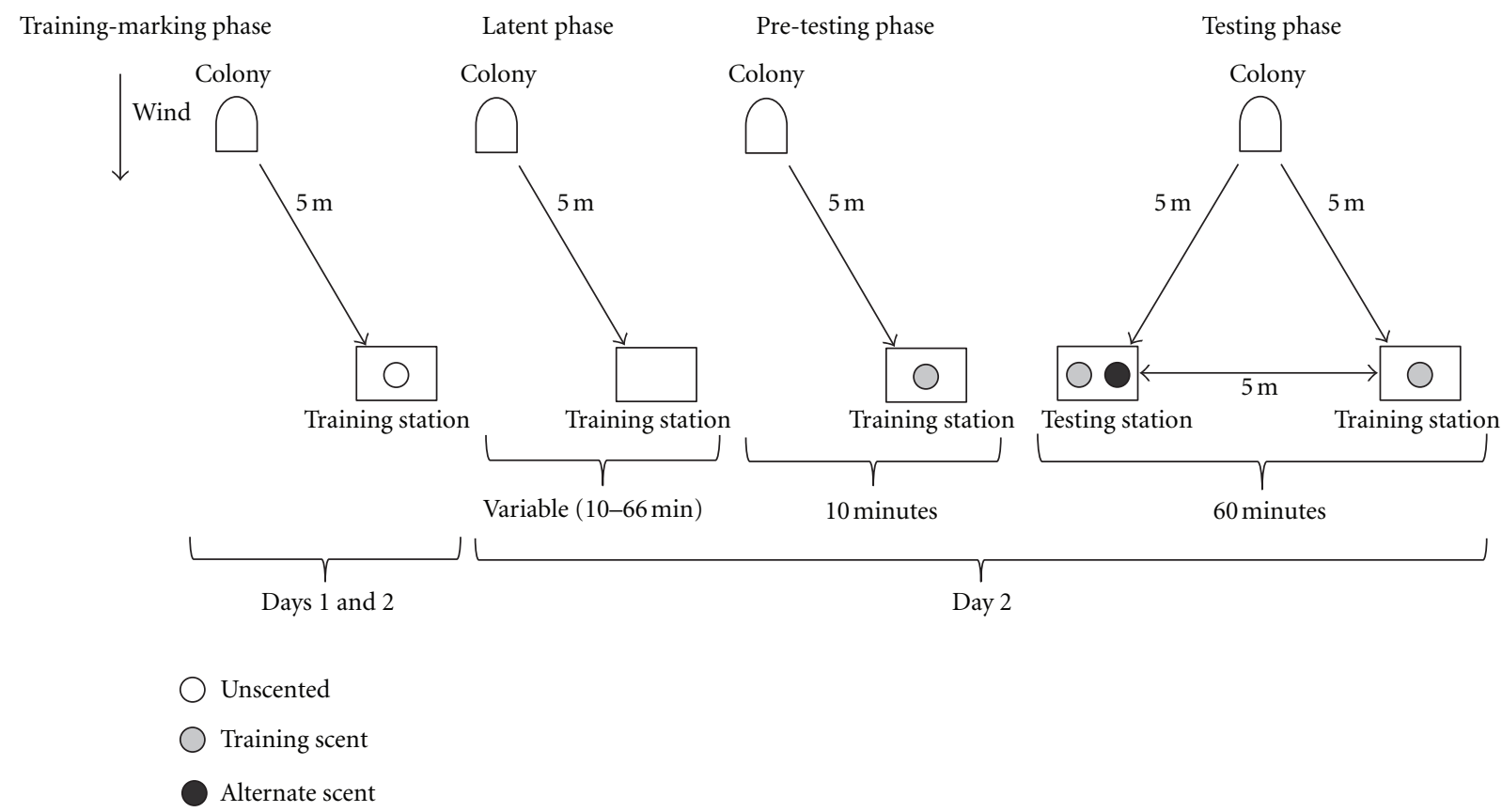

FIGURE 1: Experimental set-up and procedure. Prevailing wind direction is indicated.

testing station. For the scented solutions, extracts of pure vanilla and imitation cherry (McCormick \& Co, Inc., Hunt Valley, MD, USA) were used. Throughout the investigation, the criterion of a "visit" to a dish required that the forager land on the dish and imbibe some of the sucrose solution.

Each of seven colonies was used for one experimental trial (Table 1), consisting of a two-day training-marking phase, a latent phase, a $10 \mathrm{~min}$ pretesting phase, and a $60 \mathrm{~min}$ testing phase (Figure 1). During the training-marking phase, a large pool of foragers were trained to forage at a dish containing an unscented sucrose solution at the training station and were marked so that they could be identified during the pretesting and testing phases (Table 1). The training procedure began by placing the dish against the nest, allowing workers to feed from it, and then incrementally moving it to the final experimental location (procedure described in detail in von Frisch [35] for honey bees and Schueller et al. [7] for P. occidentalis). By the time the training dish was moved to the final location, $5 \mathrm{~m}$ downwind of the nest, many foragers were foraging from it.

For marking, each forager was caught by grasping her around the petiole with reverse-action forceps as she landed on the training dish. She was then immediately marked on the thorax with a unique color code (with Decocolor paint pens) and released. The total numbers of individuals marked during both training-marking phase days are given in Table 1. On day one marking continued until, for a period of $10 \mathrm{~min}$., all foragers arriving at the dish were already marked; in other words until there were no longer any unmarked foragers arriving. At the beginning of day two of the training-marking phase, additional unmarked foragers arrived at the dish. These, too, were marked until there were no more unmarked foragers arriving at the training station.
The ultimate location of the training station was the same for all phases of the experiment. For each colony the trainingmarking sessions were carried out on consecutive days, except for colonies 10014 and 10004, when rain intervened (Table 1).

When marking on day two was complete, the training dish was removed from the table at the training station, defining the beginning of the latent phase. With a reward no longer present, the rate of forager arrivals at the station waned. The latent phase ended when no foragers arrived for a period of $2 \mathrm{~min}$. The duration of the latent phase ranged from 10 to $66 \mathrm{~min}$ (Table 1 ). The purpose of the latent phase was to allow foraging on the unscented solution to cease before the colonies were exposed to the training scent.

At the end of the latent phase, the 10-min. pretesting phase was initiated by placing a feeder dish containing $2.0 \mathrm{M}$ sucrose solution, now scented with the training scent, atop the training table. For each colony, one scent was randomly assigned as the training scent and the other as the alternate scent (Table 1). The purpose of this phase was to allow marked foragers to resume foraging, only now they were bringing the training-scented sucrose solution to the nest. Their identities and numbers of visits to the training dish were recorded. All unmarked foragers arriving at the training dish were counted and then captured by placing a 15-dram plastic collection vial over them when they landed on the dish or table. They were placed on ice until the end of the experiment, then released. This was done to ensure that the identity of each forager arriving at the training dish was known and that no forager foraged at both the training and testing dishes.

At the end of the pretesting phase, the 60-minute testing phase was initiated by placing two dishes (henceforth 
TABLE 1: Colony information. For each colony the following are indicated: the colony identification number, dates on which foragers were marked and tested, training scent used, number of combs in the nest, number of adults present at collection (within 36 hrs of end of experiment), total number of foragers marked during the training-marking phase, and the duration of the latent phase. Colony 10028 absconded before it was collected, so it was not possible to count the number of adults on the nest.

\begin{tabular}{|c|c|c|c|c|c|c|}
\hline Colony & Dates & Training scent & Number of combs & Number of adults & Total marked & Latent phase (min) \\
\hline 10052 & June $25-26$ & cherry & 7 & 2120 & 93 & 16 \\
\hline 10048 & June $28-29$ & vanilla & 7 & 409 & 68 & 10 \\
\hline 10029 & July 2-3 & cherry & 7 & 710 & 33 & 25 \\
\hline 10014 & July 8,10 & vanilla & 6 & 1322 & 62 & 24 \\
\hline 10028 & July 11-12 & vanilla & 12 & NA & 66 & 15 \\
\hline 10018 & July 15-16 & cherry & 7 & 572 & 36 & 66 \\
\hline 10004 & July 21,23 & vanilla & 6 & 462 & 20 & 45 \\
\hline Total & & & & & 378 & \\
\hline
\end{tabular}

referred to as "testing dishes") containing a $2.0 \mathrm{M}$ sucrose solution on the table at the testing station. The testing dishes were placed $11 \mathrm{~cm}$ apart and crosswind from each other. One dish was scented with the training scent and the other with the alternate scent. All three dishes- the dish at the training station and the two at the testing station-remained in place throughout the testing phase. At the training station, marked foragers were free to make repeated visits to the dishes; their identities and the number of trips each made were recorded. At the testing station, we recorded the identity, time of arrival, and dish choice (scent and position-left or right, as seen from downwind) of each forager. After each arrival by a forager at a testing dish, the position (left versus right) of the training-scented dish was randomly assigned using a random number table. To ensure that each naïve forager arriving at the testing station made only one visit and had not previously foraged at the training dish during the pretesting and testing phases, as well as to prevent the alternate scent from being brought to the nest, all foragers, marked and unmarked, arriving at either of the testing dishes were captured in 15dram plastic collecting vials and placed on ice until the end of the experiment. Foragers were defined as "naïve" if they had not visited the training station while the scented dish was present (i.e., during the pretesting and testing phases), and so had no experience with the training scent other than potential exposure to it on the nest. By the end of the testing phase, ample sucrose solutions remained in all dishes.

Within $36 \mathrm{hrs}$ after the conclusion of its testing phase, each colony was collected after sundown by enclosing it in a plastic bag and snipping the supporting branch. The bagged nest was placed in a freezer for at least 12 hours to kill the adult wasps, and then counts were made of the number of adults present, the number of combs in the nest, the number of brood cells, the number and approximate stages of the larvae, and the number of pupae. The adults from colony 10028 absconded before collection, so the adults could not be counted.

Analysis of forager choice at the testing station was performed using a one-factor ANOVA model. We used the proportions of landings at the two dishes at the testing station, rather than absolute counts of landings, because the colonies differed in size and therefore it was not meaningful to compare absolute counts. The proportion of landings on the dishes depended both on what scent was used and whether or not the scent was the one to which the wasps were trained. In order to isolate the effect of training, particularly with a different number of trials conducted with each scent as the training scent, our analysis had to account for both factors. To do so, we define $Y_{i j}$ as the proportion of landings on the dish containing vanilla, whether or not it was the training scent. The model underlying our analysis can be written as

$$
Y_{i j}=\mu+T_{i}+e_{i j},
$$

where $i=1,2$ corresponds to the training scent ( $1=$ vanilla, $2=$ cherry); $j$ indexes the trial number for each level of $i$; $\mu$ is the overall mean; $T_{i}$ is the treatment effect; $e_{i j}$ is the random error. We used a weighted ANOVA (with weights proportional to total number of arrivals at the testing station), recognizing that a trial with more landings will result in a more precise measure of the proportion. Because the response variable is a proportion, we used the "arcsine square-root" transformation to achieve homogeneous variance [36].

We predicted that if marked foragers are communicating directional information back at the nest, significantly more naïve foragers would arrive at the training station. A paired $t$-test was used to compare the number of naïve foragers arriving at the training station to the number arriving at the testing station.

Our analysis of foraging-rate patterns during the pretesting and testing phases explored the relationships between various pairs of the variables: total forager arrivals, average number of trips per forager, number of marked foragers at the training stations, total number of adults, number of larvae, and latent phase. Where appropriate, simple linear regression analyses were performed to quantify the relationships.

Five additional experimental trials were performed, but results were excluded from the forager-choice analysis and/or foraging-rate analyses. For three of these colonies, the total number of arrivals at the testing station was one or none, and thus the colonies provided insufficient data for either analysis. During another trial, the marks on the majority of 
TABLE 2: Arrivals at the training and testing stations during the pretesting and testing phases. Column 2: number of marked foragers making one or more trips to the training station. Columns 3-11: numbers of arrivals. Only the marked foragers visiting the training station (column 3 ) were allowed to make repeated visits. All others were captured and held on ice upon arrival; therefore, columns 4 and 6-11 represent both numbers of arrivals and numbers of individuals arriving.

\begin{tabular}{|c|c|c|c|c|c|c|c|c|c|c|}
\hline \multirow[b]{4}{*}{ (1) $\mathrm{Col}(2)$} & \multicolumn{4}{|c|}{ Training station } & \multirow{2}{*}{\multicolumn{6}{|c|}{$\begin{array}{c}\text { Testing station } \\
\text { Number of arrivals }\end{array}$}} \\
\hline & \multirow{2}{*}{\multicolumn{4}{|c|}{$\begin{array}{c}\text { Number of arrivals } \\
\text { Training scent }\end{array}$}} & & & & & & \\
\hline & & & & & \multicolumn{3}{|c|}{ Training scent } & \multicolumn{3}{|c|}{ Alternative scent } \\
\hline & Number mk'd indiv & (3) Mk'd & (4) Unmk'd & (5) Total & (6) Mk'd & (7) Unmk'd & (8) Ttl & (9) Mk'd & (10) Unmk'd & (11) $\mathrm{Ttl}$ \\
\hline 04 & 6 & 197 & 16 & 213 & 3 & 0 & 3 & 1 & 0 & 1 \\
\hline 18 & 7 & 360 & 8 & 368 & 1 & 6 & 7 & 1 & 1 & 2 \\
\hline 29 & 8 & 270 & 3 & 273 & 0 & 3 & 3 & 0 & 0 & 0 \\
\hline 14 & 11 & 204 & 5 & 209 & 1 & 2 & 3 & 1 & 1 & 2 \\
\hline 28 & 14 & 266 & 16 & 282 & 3 & 3 & 6 & 0 & 3 & 3 \\
\hline 52 & 22 & 185 & 1 & 186 & 3 & 6 & 9 & 2 & 4 & 6 \\
\hline 48 & 33 & 179 & 10 & 189 & 1 & 3 & 4 & 0 & 3 & 3 \\
\hline $\mathrm{Ttl}$ & 101 & 1661 & 59 & 1720 & 12 & 23 & 35 & 5 & 12 & 17 \\
\hline
\end{tabular}

TABLE 3: The relationship between the total number of arrivals to the training station and the average number of foraging trips/forager to the training station, the number of adults in colony, the number of marked foragers foraging at the training station, the number of larvae in the colony, and the duration of the latent phase, using a simple regression analysis.

\begin{tabular}{|c|c|c|c|c|}
\hline Source & Slope & Adjusted $R^{2}$ & $d f$ & $P$ \\
\hline Average number of trips/forager & 3.71 & 0.84 & 6 & 0.0009 \\
\hline Number of adults & 0.030 & -0.13 & 5 & 0.594 \\
\hline Number of marked foragers foraging at training station & -5.56 & 0.27 & 6 & 0.108 \\
\hline Number of larvae & 0.044 & -0.023 & 6 & 0.395 \\
\hline Latent phase (min) & 2.22 & 0.34 & 5 & 0.095 \\
\hline
\end{tabular}

foragers arriving at the testing station indicated that they were members of a nearby, newly relocated swarm and not from the colony being tested. The fifth trial (colony 10052) was excluded because its results appeared to have been biased by a previous trial using the same colony. This colony was tested on June 26 with cherry as the training scent and again on July 1 with vanilla as the training scent. Sixty-seven percent of the foragers coming to the testing dishes on July 1 chose the cherry-scented solution. Since $P$. occidentalis colonies store nectar $[6,27]$, it was likely that colony members had been exposed to the scent of cherry for five days and thus were apt to search for the cherry rather than the newly introduced vanilla. Although this trial was not used for the forager-choice or latent phase analysis, it was included in foraging-rate analysis because nectar storage is not expected to affect foraging rates. Colony 10028 was excluded from regression analyses involving total numbers of adults because the colony members absconded before it could be collected and censused.

\section{Results}

3.1. Training Station. One-hundred-one marked foragers from the seven colonies made a total of 1,661 trips to the training dish during the pretesting and testing phases $($ mean $=16.4, \mathrm{SD}=17.3, \mathrm{max} / \mathrm{min}=65 / 1)$ (Figure 3;
TABLE 4: Relationship between the total number of foraging trips to the training dish/forager and latent phase ( $\mathrm{min}$ ), the number of adults in the colony, and the number of larvae in the colony, using a simple regression analysis.

\begin{tabular}{lcccc}
\hline Source & Slope & Adjusted $R^{2}$ & $d f$ & $P$ \\
\hline Latent phase (min) & 0.74 & 0.80 & 5 & 0.0039 \\
Number of adults & 0.0050 & -0.17 & 5 & 0.726 \\
Number of larvae & 0.012 & -.029 & 6 & 0.404 \\
\hline
\end{tabular}

Table 2). In addition, fifty-nine unmarked foragers arrived at the training dish during the pretesting and testing phases (Table 2).

The distributions of the numbers of foraging trips made by marked foragers from each nest to the training station for each colony during the pretesting and testing phases are shown in Figure 3. The total number of arrivals to the training station, a measure of colony foraging effort, is correlated with the average number of foraging trips/forager, but not with the number of marked foragers foraging at the training dish, the total number of adults in the colony, or the number of brood in the second larval instar and above (Figure 4, Table 3). The number of foraging trips to the training dish per forager is correlated with the duration of the latent phase, but not the number of adults in the colony, or the number of larvae in the colony (Figure 5, Table 4). 


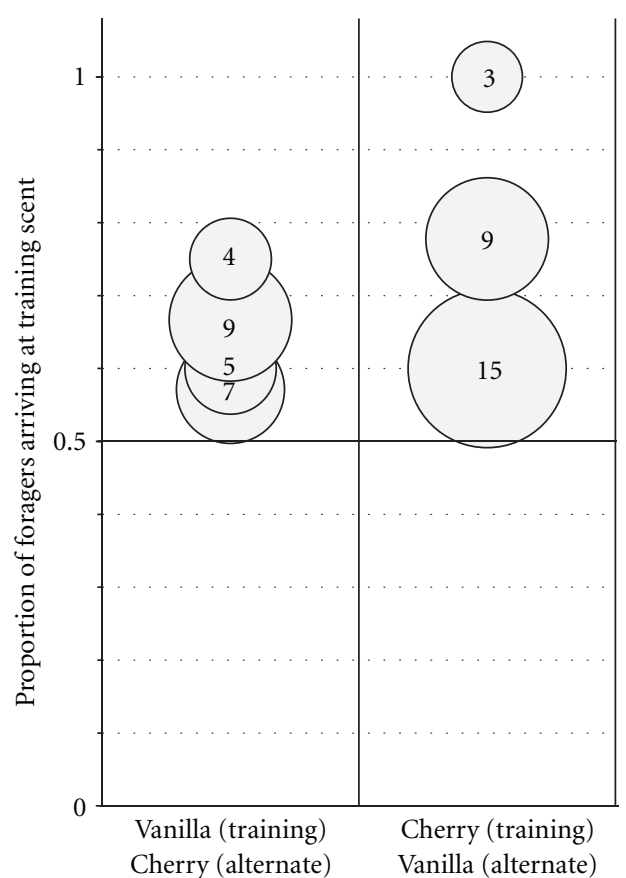

Figure 2: Arrivals at the testing station. Training and alternate scents are indicated. Each circle represents one colony. The center of the circle indicates the proportion arriving at the training scented dish for the colony. The area of the circle is proportional to the total numbers of unmarked foragers arriving at either of the dishes at the testing station; this number is indicated by the number inside the circle.

3.2. Testing Station. Fifty-two foragers from the seven colonies arrived at the one or the other of the two testing dishes during the testing phase (Table 2). Of those, thirtyfive were unmarked, so had not previously foraged from any of the dishes (Table 2). Across all colonies, two-thirds of the foragers that arrived at the testing station chose the training scent over the alternate scent F1, $5=7.32, N=7, P=0.0425$ (Figure 2).

The average time between forager arrivals at the testing station was 373 seconds, $\mathrm{SD}=531, \mathrm{max} / \mathrm{min}=2741 / 21 \mathrm{sec}-$ onds, $N=45$ ( 7 foragers were the first arrivals so could not be used for this analysis). None arrived while another was at the station.

The number of unmarked foragers arriving at the training station (59) did not differ significantly from the total number of naïve foragers arriving at the testing station (52) (paired $t$-test: $d f=6, T=-0.327, P=0.755$ ), or the number of unmarked foragers arriving at the testing station (35) (paired $t$-test: $d f=6, T=-1.149, P=0.294$ ). No marked forager foraged at both the training and testing stations during the testing phase. Only 118 (31\%) of the 378 foragers that were marked during the training-marking phase arrived at the stations during the pretesting and testing phases (Tables 1 and 2).

\section{Discussion}

In this investigation, naïve foragers learned the scent of a carbohydrate solution brought to the nest by successful foragers and used the food-scent cue to locate a sucrose solution at a novel location off nest. Foragers brought the scented sucrose solution to the nest in amounts many times smaller than those added artificially to nests in a previous study [7] and represented a naturally attainable rate of food influx into the nest.

Newly-activated foragers arrived in equal numbers at the training and testing stations, suggesting that these foragers searched for a familiar resource off nest without obtaining directional information at the nest. This result also fails to provide any evidence that $P$. occidentalis lays scent-marks to rich food sources, as it does to new nest sites $[26,33,34]$. Because there was never a wasp present when a forager arrived at the testing station, foragers could not have been using local enhancement to make a choice between the dishes. We conclude, therefore, that naïve foragers relied upon olfactory cues to locate the scented solution off nest. This is the first demonstration that $P$. occidentalis foragers are activated to visit a novel feeder location in response to foragers bringing a scented resource to the nest, without having been trained to arrive at that specific location $[6,7]$ or using local enhancement cues [6].

The strength of volatiles characterizing natural carbohydrate sources utilized by $P$. occidentalis no doubt varies. Honeydew and extrafloral nectars may provide relatively weak olfactory cues, whereas floral nectars and ripe and rotting fruits no doubt provide strong cues that are detectable from some distance downwind [37]. It is likely that both olfactory cues emanating from a fruit and the visual cue of conspecifics already foraging on it are both utilized by searching foragers, but their relative importance may vary depending on a number of variables, including wind strength and direction and line-of-sight visual distance.

How effective were the active foragers in bringing naïve wasps to the resources? Across all colonies, the 1,661 trips to the training dishes by marked foragers resulted in 111 foragers arriving at the training and testing stations. Thus, it took $1,661 / 111=15.0$ forager loads of $2.0 \mathrm{M}$ sucrose to bring each new forager to the resources. By comparison, when Taylor et al. [30] applied $4 \mathrm{~mL}$, or approximately 600 forager loads $[30,31]$, of the same resource (2.0 M sucrose) directly to the nest, it resulted in approximately 36 additional foragers exiting the nest. By the same calculation, it required $600 / 36=16.7$ forager load equivalents to stimulate one extra departure. Taylor et al. [30] counted new foragers as they left the nest, whereas in our investigation the new foragers were counted as they arrived at the resources $5 \mathrm{~m}$ downwind from the nest. Since it is unlikely that all naïve foragers departing in search of the scented resource experienced in the nest succeed in finding the resource, the fact that the two figures are so similar suggests that foragers returning with a rich resource (this study) may provide some form of alerting signal that stimulates more to leave the nest than would in response to the resource alone [30]. Although this comparison of the 

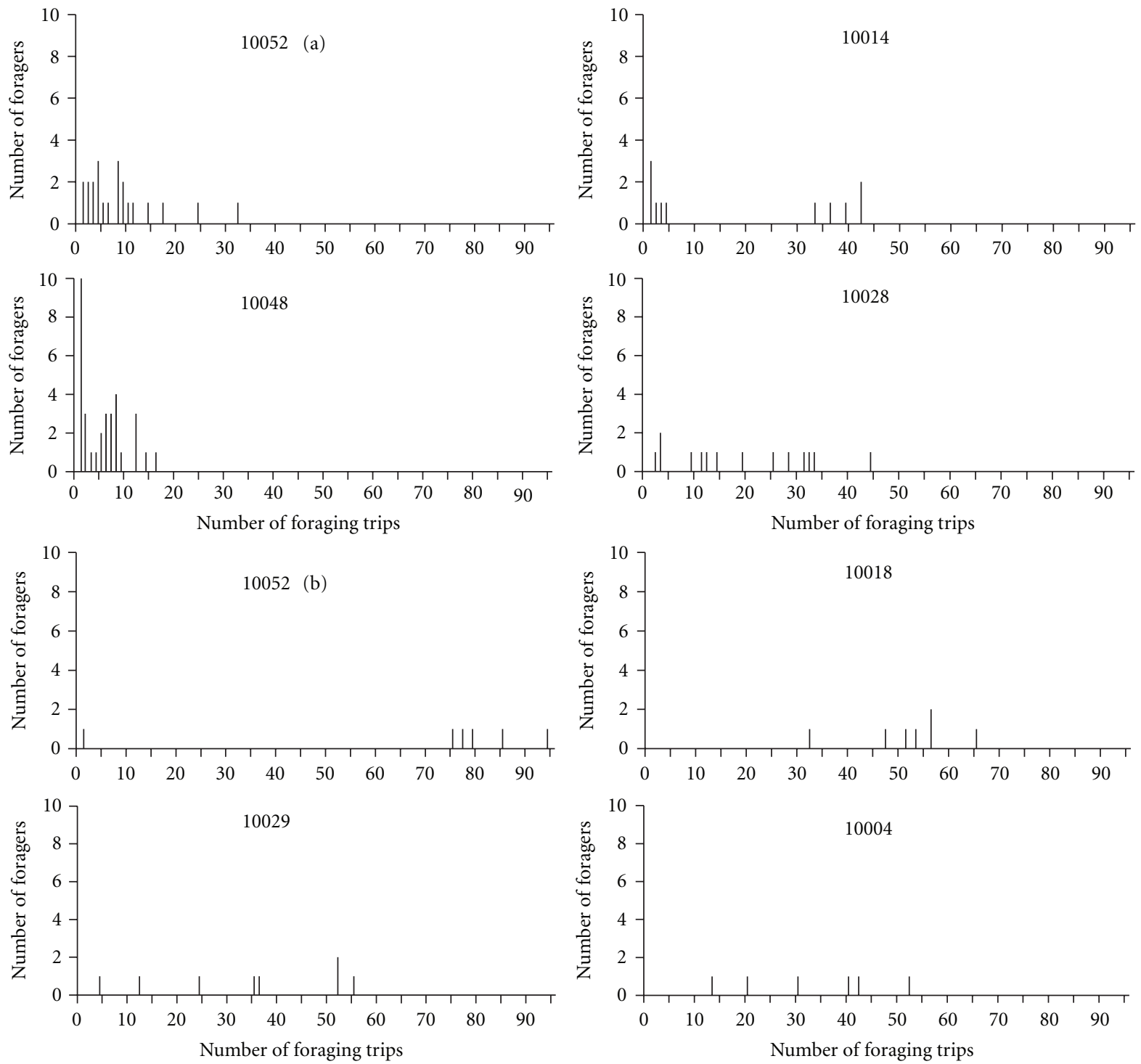

FIGURE 3: Distribution of numbers of foraging trips each marked forager made to the training station during both the pretesting and testing phases. The $x$-axis represents the number of trips by each forager and the $y$-axis represents the number of foragers making the indicated number of trips. 10052 (a) refers to colony 10052 when it was tested on June 26 and 10052 (b) refers to colony 10052 when tested again on July 1.

results of the two studies is rather crude, it is nonetheless worth making for the experiments it may suggest.

Foraging effort, measured as the total number of foraging trips made by marked foragers, did not correlate with colony demand, as measured by either the number of adults or the number of larvae in the colony. This result contrasts with that of Howard and Jeanne [28], who found a strong correlation between foraging rate and colony demand in the same species. The difference may be attributable to differences in the methods used. Howard and Jeanne measured ongoing foraging rates in unmanipulated colonies [28], whereas we trained a set of foragers to make repeated visits to a rich resource. Foraging rates vary tremendously among individuals [38] for reasons that are not well understood. The overall rate at which each colony exploited the resource may have been a function of the individual rates of those foragers in the subset that happened to have been trained to our dishes, independently of the demands of the colony.

Given that colony-level foraging effort bore no relation to colony demand, nor to the number of active foragers in the colony, measured as the number of marked individuals arriving at the testing station, one might then expect a positive correlation between the number of foragers making repeat visits in each colony and the total number of visits to the dishes made by each colony. In contrast to expectation, the relationship trended negative, although not quite significantly so. Although the regression of total trips per colony on the number of active foragers was not significant, there was a positive correlation between total trips and the average number of foraging trips/forager for each colony. 


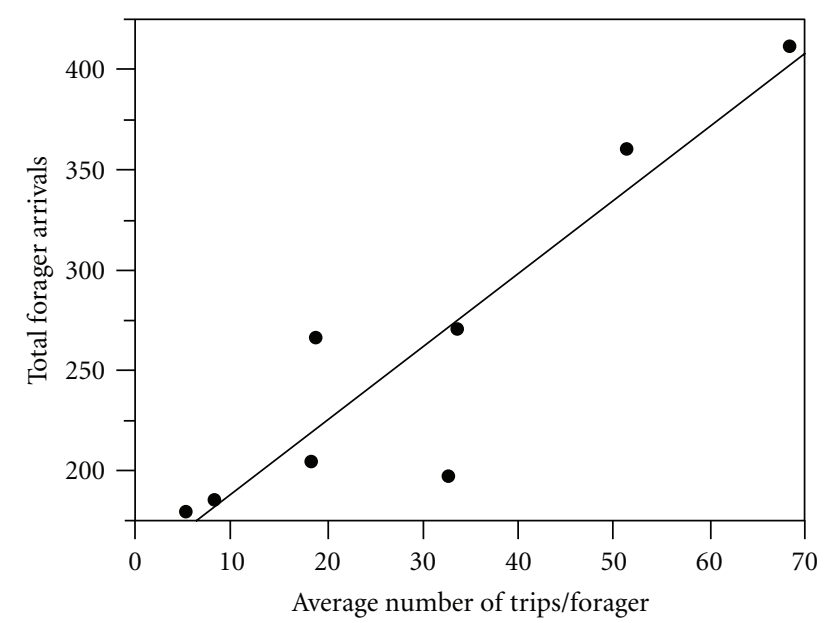

Figure 4: The total number of arrivals of marked foragers, a measure of colony-wide foraging effort, at the training station during the pretesting and testing phases as a function of the average number of trips per forager.

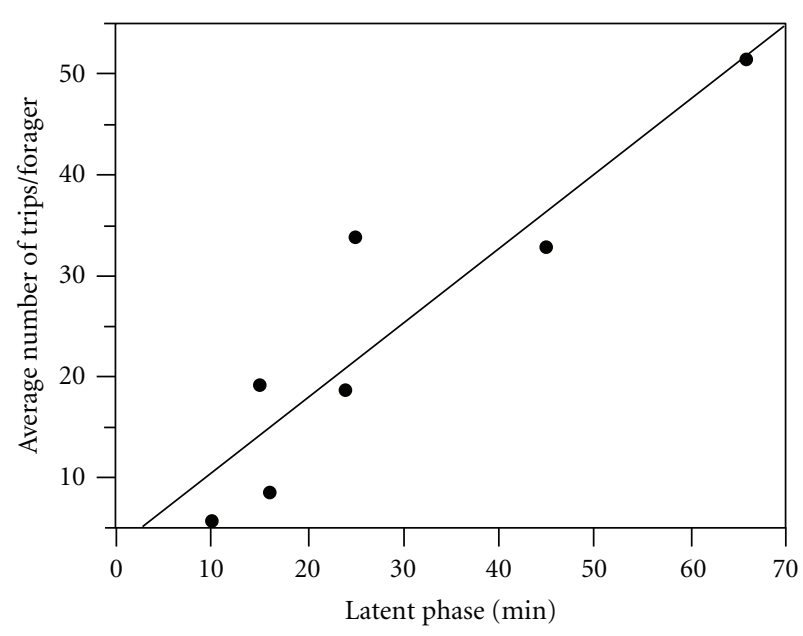

FIGURE 5: Average number of trips/forager for marked foragers arriving at the training station during the pretesting and testing phases as a function of the duration of the latent phase.

That is, in the colonies that employed the largest number of foragers, those foragers performed the fewest foraging trips, on average. In fact, this relationship was so strong that in colonies employing the fewest foragers, not only did each forager make more trips than in large-group colonies, but the total number of trips made by these colonies was almost double those in the large-group colonies. This is a counterintuitive pattern. A reasonable a priori assumption would be that samples of 6-33 foragers (Table 2) would have similar mean per-capita foraging rates, and therefore that the overall number of trips made by those samples would be in direct relation to the number of foragers in the sample. That this was not the case suggests the existence of some kind of group-size-related dynamic.

One possibility for the observed pattern is that, compared to colonies with small numbers of foragers, in colonies with a large number of foragers returning to the nest, each forager received a less-than-enthusiastic reception by nectar receivers, or a longer unloading-delay, causing them to wait longer before making the next trip. Another possibility is that colonies with the highest overall foraging rates had a higher proportion of "elites" actively foraging than the colonies with lower overall rates. Elite foragers make a disproportionate number of trips [39]. Although the presence of elite nectar foragers has not been documented in P. occidentalis, they have been shown to exist in Vespula germanica [39], and it has been shown that some $P$. occidentalis make disproportionately more of the foraging trips for nest building materials [40].

Interestingly, the number of foraging trips per forager was also positively correlated with the length of the latent phase, which may be a reflection of the average persistence and foraging rate of the colony's foragers. During the latent phase, the training dish was removed from the training station, disrupting any foraging reinforcement until foragers were no longer arriving at the station. Colonies with the most active and persistent foragers would, therefore, have the longest latent phase. This pattern might also be explained by different proportions of actively foraging elites across colonies. If elite foragers, in general, not only make relatively more foraging trips than nonelites, but also tend to persist longer at the task [40], then one would predict that the latent phase for colonies employing more elites would be longer than those with fewer active elites, the pattern observed during this investigation.

Results of the present investigation demonstrate that the social wasp $P$. occidentalis uses a cue-based form of food recruitment whereby the arrival in the nest of foragers with a rich, scented carbohydrate resource induces naïve nestmates to forage at an off nest resource with the same scent. Many groups of social insects employ this simple mechanism [41]. In choice experiments, naïve Vespula vulgaris and $V$. germanica foragers chose resources with the same scent as the ones that were brought to the nest by successful foragers $[22,42]$. When a scented carbohydrate solution was added directly to the nest, naïve foragers also preferentially chose the resources with the same scent off nest [23]. Similarly, bumble bee (Bombus terrestris) foragers preferentially chose resources with the same scent as those stored in honey pots inside the colony $[17,41]$. Honey bee foragers use scent cues experienced at the nest, in addition to the waggle dance, to help locate food sources off nest $[4,15,35,43-45]$. Cue-based recruitment to food sources has the advantage of allowing foragers to home in on particularly profitable resources, thus, expending less energy than independent searching would require $[5,45]$. Recruitment is especially advantageous for species such as $P$. occidentalis that live in the tropics, where resources are more patchily and ephemerally distributed than in temperate regions $[45,46]$.

In light of the results from this and several other recent investigations, a good picture of carbohydrate foraging and recruitment for $P$. occidentalis can now be drawn. Foragers learn to associate visual and olfactory cues with a carbohydrate resource and use these to aid them in relocating the resource on return visits $[7,47]$. They do not mark 
the resource with a pheromone signal [31]. Meanwhile, the influx of carbohydrates from successful foragers incites inactive foragers to leave the nest and search off nest. They obtain no directional information from the successful foragers, but they do learn the olfactory cues of the food brought to the nest and search for a resource with the same scent $([6,30]$, present investigation). Local enhancement is a second site-based cue that attracts nestmates visually to a specific resource [6]. Both kinds of cues are likely to shorten search time, thereby decreasing energetic expenditures and mortality, by allowing foragers to home in on high quality resources more quickly than by uninformed random searching ([7, 30, 48, 49], present investigation). This mechanism of activating previously inactive foragers may aid in rapid exploitation of resources when they become available [30].

One of the lingering questions about foraging in $P$. occidentalis is whether or not signals play a role in the recruitment process. Taylor et al. [30] observed an increase in rapid running across the envelope when a carbohydrate resource was added to the nest. This behavior was described as being much like the wing fanning and animated running signals used by successful Bombus spp. foragers that induce nestmates to begin foraging [50-53] and may indicate that $P$. occidentalis uses an alerting signal as well as a cue-mediated recruitment mechanism [30]. This has yet to be confirmed [30] but, if used, may serve to enhance the efficacy of cuebased recruitment by activating more individuals than is possible via cues alone.

\section{Acknowledgments}

This project would not have been possible without the generosity of the Hagnauer family and the staff at Hacienda La Pacifica in allowing the authors to conduct research on their properties. The authors give sincere thanks to Evan Brus for assistance in the field. Erik Nordheim offered valuable guidance with statistics, and Benjamin Taylor provided helpful comments and suggestions on the paper. Research was supported by the Zoology Department, University of Wisconsin-Madison. T. I. Schueller was supported by a Dickie Family Sauk County Educational Award. Neither T. I. Schueller or R. L. Jeanne has a financial relationship with Decocolor, Tanglefoot, Plexiglas, or McCormick \& Co. or any other commercial entity referenced in this paper.

\section{References}

[1] E. O. Wilson, The Insect Societies, The Belknap Press of Harvard University Press, Cambridge, Mass, USA, 1971.

[2] M. Beekman and A. Dussutour, "How to tell your mates: costs and benefits of different recruitment mechanisms," in Food Exploitation by Social Insects: Ecological, Behavioral, and Theoretical Approaches, S. Jarau and M. Hrncir, Eds., pp. 115134, CRC Press, Boca Raton, Fla, USA, 2009.

[3] C. Detrain and J. Deneubourg, "Social cues and adaptive foraging social cues and adaptive foraging strategies in ants," in Food Exploitation by Social Insects: Ecological, Behavioral, and Theoretical Approaches, S. Jarau and M. Hrncir, Eds., pp. 2951, CRC Press, Boca Raton, Fla, USA, 2009.
[4] C. Grüter and F. L. W. Ratnieks, "Honeybee foragers increase the use of waggle dance information when private information becomes unrewarding," Animal Behaviour, vol. 81, no. 5, pp. 949-954, 2011.

[5] M. Lachmann, G. Sella, and E. Jablonka, "On the advantages of information sharing," Proceedings of the Royal Society B: Biological Sciences, vol. 267, no. 1450, pp. 1287-1293, 2000.

[6] M. Hrncir, S. Mateus, and F. S. Nascimento, "Exploitation of carbohydrate food sources in Polybia occidentalis: social cues influence foraging decisions in swarm-founding wasps," Behavioral Ecology and Sociobiology, vol. 61, no. 6, pp. 975983, 2007.

[7] T. I. Schueller, E. V. Nordheim, B. J. Taylor, and R. L. Jeanne, "The cues have it; nest-based, cue-mediated recruitment to carbohydrate resources in a swarm-founding social wasp," Naturwissenschaften, vol. 97, no. 11, pp. 1017-1022, 2010.

[8] D. Otte, "Effects and functions in the evolution of signaling systems," Annual Review of Ecology and Systematics, vol. 5, pp. 385-417, 1974.

[9] J. E. Lloyd, "Bioluminescence and communication in insects," Annual Review of Entomology, vol. 28, pp. 131-160, 1983.

[10] M. S. Dawkins, Unravelling Animal Behaviour, John Wiley \& Sons, New York, NY, USA, 1995.

[11] T. D. Seeley, "Thoughts on information and integration in honey bee colonies," Apidologie, vol. 29, no. 1-2, pp. 67-80, 1998.

[12] C. Anderson and D. W. McShea, "Individual versus social complexity, with particular reference to ant colonies," Biological Reviews of the Cambridge Philosophical Society, vol. 76, no. 2, pp. 211-237, 2001.

[13] R. L. Jeanne, "Social complexity in the Hymenoptera, with special attention to the wasps," in Genes, Behaviors and Evolution of Social Insects, T. Kikuchi, N. Azuma, and S. Higashi, Eds., pp. 81-130, Hokkaido University Press, Sapporo, Japan, 2003.

[14] R. L. Jeanne and B. J. Taylor, "Individual and social foraging in social wasps," in Food Exploitation In Social Insects: Ecological Behavioral, and Theoretical Approaches, S. Jarau and M. Hrncir, Eds., pp. 53-79, CRC Press, Boca Raton, Fla, USA, 2009.

[15] W. M. Farina, C. Grüater, and P. C. Díaz, "Social learning of floral odours inside the honeybee hive," Proceedings of the Royal Society B: Biological Sciences, vol. 272, no. 1575, pp. 1923-1928, 2005.

[16] J. C. Biesmeijer and E. J. Slaa, "Information flow and organization of stingless bee foraging," Apidologie, vol. 35, no. 2, pp. 143-157, 2004.

[17] A. Dornhaus and L. Chittka, "Information flow and regulation of foraging activity in bumble bees (Bombus spp.)," Apidologie, vol. 35, no. 2, pp. 183-192, 2004.

[18] J. C. Nieh, "Recruitment communication in stingless bees (Hymenoptera, Apidae, Meliponini)," Apidologie, vol. 35, no. 2, pp. 159-182, 2004.

[19] M. S. Balbuena, J. Molinas, and W. M. Farina, "Honeybee recruitment to scented food sources: correlations between inhive social interactions and foraging decisions," Behavioral Ecology and Sociobiology, vol. 66, pp. 445-452, 2012.

[20] R. L. Jeanne, J. H. Hunt, and M. G. Keeping, "Foraging in social wasps: Agelaia lacks recruitment to food (Hymenoptera: Vespidae)," Journal - Kansas Entomological Society, vol. 68, no. 3, pp. 279-289, 1995. 
[21] M. R. Richter, "Hunting social wasp interactions: influence of prey size, arrival order, and wasp species," Ecology, vol. 71, no. 3, pp. 1018-1030, 1990.

[22] S. L. Overmyer and R. L. Jeanne, "Recruitment to food by the German yellowjacket, Vespula germanica," Behavioral Ecology and Sociobiology, vol. 42, no. 1, pp. 17-21, 1998.

[23] J. M. Jandt and R. L. Jeanne, "German yellowjacket (Vespula germanica) foragers use odors inside the nest to find carbohydrate food sources," Ethology, vol. 111, no. 7, pp. 641-651, 2005.

[24] M. Ono, T. Igarashi, E. Ohno, and M. Sasaki, "Unusual thermal defence by a honeybee against mass attack by hornets," Nature, vol. 377, no. 6547, pp. 334-336, 1995.

[25] E. O. Wilson, Success and Dominance in Ecosystems: the Case of the Social Insects, Ecology Institute, Oldendorf/Luhe, Germany, 1990.

[26] R. L. Jeanne, "The swarm-founding Polistinae," in The Social Biology of Wasps, K. G. Ross and R. W. Matthews, Eds., pp. 191-231, Cornell University Press, Ithaca, NY, USA, 1991.

[27] J. H. Hunt, A. M. Rossi, N. J. Holmberg, S. R. Smith, and W. R. Sherman, "Nutrients in social wasp (Hymenoptera: Vespidae, Polistinae) honey," Annals of the Entomological Society of America, vol. 91, no. 4, pp. 466-472, 1998.

[28] K. J. Howard and R. L. Jeanne, "Rates of brood development in a social wasp: Effects of colony size and parasite infection," Insectes Sociaux, vol. 51, no. 2, pp. 179-185, 2004.

[29] B. J. Taylor, E. V. Nordheim, T. I. Schueller, and R. L. Jeanne, "Recruitment in swarm-founding wasps: Polybia occidentalis does not actively scent-mark carbohydrate food sources," Psyche, vol. 2011, Article ID 378576, 7 pages, 2011.

[30] B. J. Taylor, E. J. Brus, and R. L. Jeanne, "Introduction of a scented carbohydrate resource into the nest increases departure rate in Polybia occidentalis," Insectes Sociaux, vol. 59, no. 2, pp. 151-157, 2012.

[31] R. L. Jeanne, "The organization of work in Polybia occidentalis: costs and benefits of specialization in a social wasp," Behavioral Ecology and Sociobiology, vol. 19, no. 5, pp. 333-341, 1986.

[32] K. J. Howard and R. L. Jeanne, "Shifting foraging strategies in colonies of the social wasp Polybia occidentalis (Hymenoptera, Vespidae)," Behavioral Ecology and Sociobiology, vol. 57, no. 5, pp. 481-489, 2005.

[33] R. L. Jeanne, "Chemical communication during swarm emigration in the social wasp Polybia sericea (Olivier)," Animal Behaviour, vol. 29, no. 1, pp. 102-113, 1981.

[34] P. J. Sonnentag and R. L. Jeanne, "Initiation of abscondingswarm emigration in the social wasp Polybia occidentalis," Journal of Insect Science, vol. 9, article no. 11, 2009.

[35] K. von Frisch, The Dance Language and Orientation of Bees, The Belknap Press of Harvard University Press, Cambridge, Mass, USA, 1967.

[36] P. N. Lehner, Handbook of Ethological Methods, Cambridge University Press, Cambridge, NY, USA, 1996.

[37] N. Dudareva, F. Negre, D. A. Nagegowda, and I. Orlova, "Plant volatiles: Recent advances and future perspectives," Critical Reviews in Plant Sciences, vol. 25, no. 5, pp. 417-440, 2006.

[38] R. L. Jeanne, "Polyethism," in The Social Biology of Wasps, K. G. Ross and R. W. Matthews, Eds., pp. 389-425, Cornell University Press, Ithaca, NY, USA, 1991.

[39] C. R. Hurd, E. V. Nordheim, and R. L. Jeanne, "Elite workers and the colony-level pattern of labor division in the yellow jacket wasp, Vespula germanica," Behaviour, vol. 140, no. 7, pp. 827-845, 2003.

[40] S. O'Donnell and R. L. Jeanne, "Forager specialization and the control of nest repair in Polybia occidentalis Olivier (Hymenoptera : Vespidae)," Behavioral Ecology and Sociobiology, vol. 27, no. 5, pp. 359-364, 1990.

[41] A. Dornhaus and L. Chittka, "Evolutionary origins of bee dances," Nature, vol. 401, no. 6748, p. 38, 1999.

[42] U. Maschwitz, W. Beier, I. Dietrich, and W. Keidel, "Futterverständigung bei Wespen der Gattung Paravespula," Die Naturwissenschaften, vol. 61, no. 11, p. 506, 1974.

[43] J. Reinhard, M. V. Srinivasan, and S. Zhang, "Scent-triggered navigation in honeybees," Nature, vol. 427, no. 6973, p. 411, 2004.

[44] J. Reinhard and M. V. Srinivasan, "The role of scents in honey bee foraging and recruitment," in Food Exploitation In Social Insects Ecological Behavioral, and Theoretical Approaches, S. Jarau and M. Hrncir, Eds., pp. 165-182, CRC Press, Boca Raton, Fla, USA, 2009.

[45] A. Dornhaus, F. Klügl, C. Oechslein, F. Puppe, and L. Chittka, "Benefits of recruitment in honey bees: Effects of ecology and colony size in an individual-based model," Behavioral Ecology, vol. 17, no. 3, pp. 336-344, 2006.

[46] A. Dornhaus and L. Chittka, "Why do honey bees dance?" Behavioral Ecology and Sociobiology, vol. 55, no. 4, pp. 395401, 2004

[47] S. Shafir, "Color discrimination conditioning of a wasp, Polybia occidentalis (Hymenoptera: Vespidae)," Biotropica, vol. 28, no. 2, pp. 243-251, 1996.

[48] P. Schmid-Hempel and T. Wolf, "Foraging effort and life span of workers in a social insect," Journal of Animal Ecology, vol. 57, no. 2, pp. 509-521, 1988.

[49] S. O’Donnell and R. L. Jeanne, "Lifelong patterns of forager behaviour in a tropical swarm-founding wasp: effects of specialization and activity level on longevity," Animal Behaviour, vol. 44, no. 6, pp. 1021-1027, 1992.

[50] A. Dornhaus and L. Chittka, "Food alert in bumblebees (Bombus terrestris): Possible mechanisms and evolutionary implications," Behavioral Ecology and Sociobiology, vol. 50, no. 6, pp. 570-576, 2001.

[51] A. Dornhaus and L. Chittka, "Bumble bees (Bombus terrestris) store both food and information in honeypots," Behavioral Ecology, vol. 16, no. 3, pp. 661-666, 2005.

[52] A. Dornhaus, A. Brockmann, and L. Chittka, "Bumble bees alert to food with pheromone from tergal gland," Journal of Comparative Physiology A: Neuroethology, Sensory, Neural, and Behavioral Physiology, vol. 189, no. 1, pp. 47-51, 2003.

[53] M. Molet, L. Chittka, R. J. Stelzer, S. Streit, and N. E. Raine, "Colony nutritional status modulates worker responses to foraging recruitment pheromone in the bumblebee Bombus terrestris," Behavioral Ecology and Sociobiology, vol. 62, no. 12, pp. 1919-1926, 2008. 

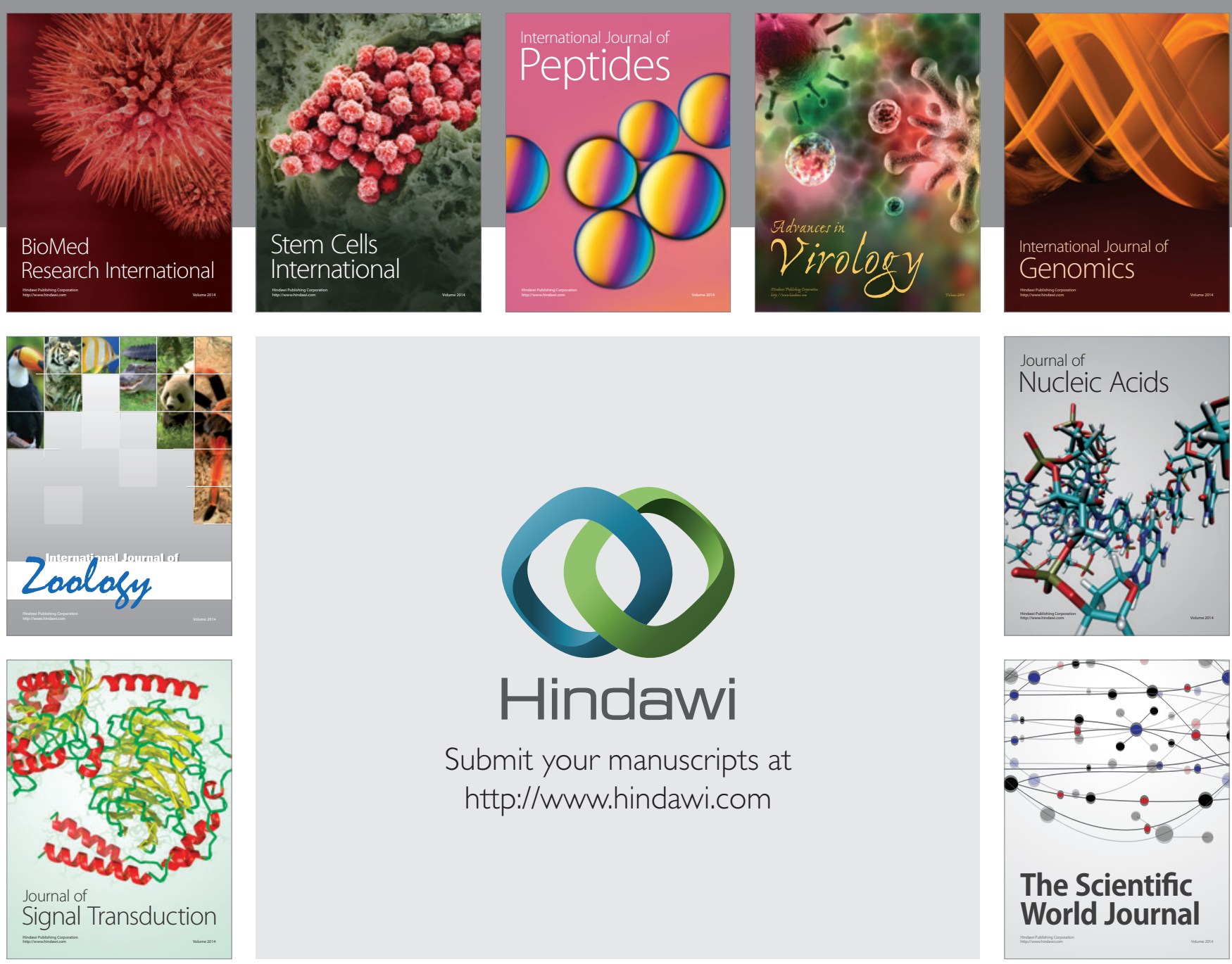

Submit your manuscripts at

http://www.hindawi.com
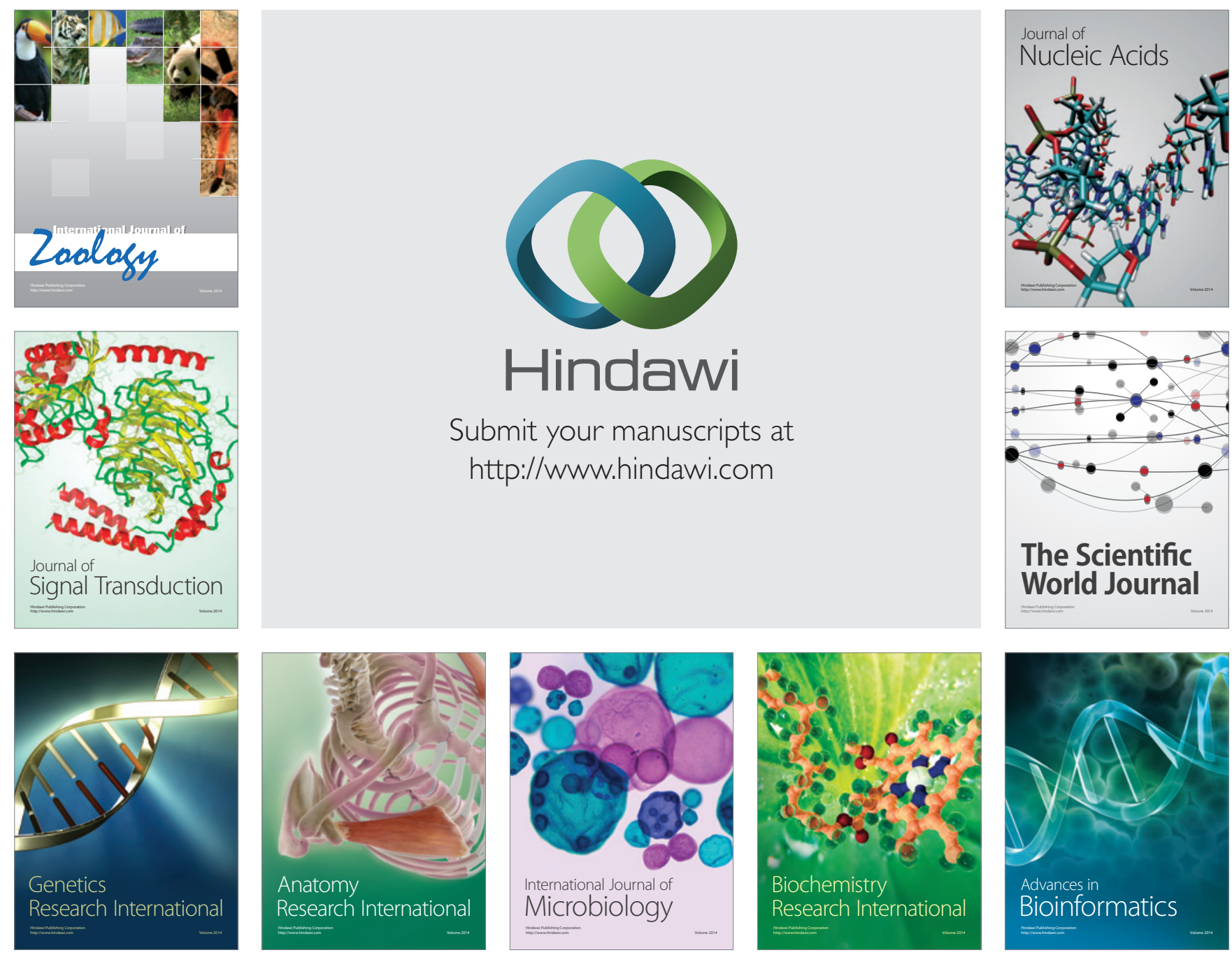

The Scientific World Journal
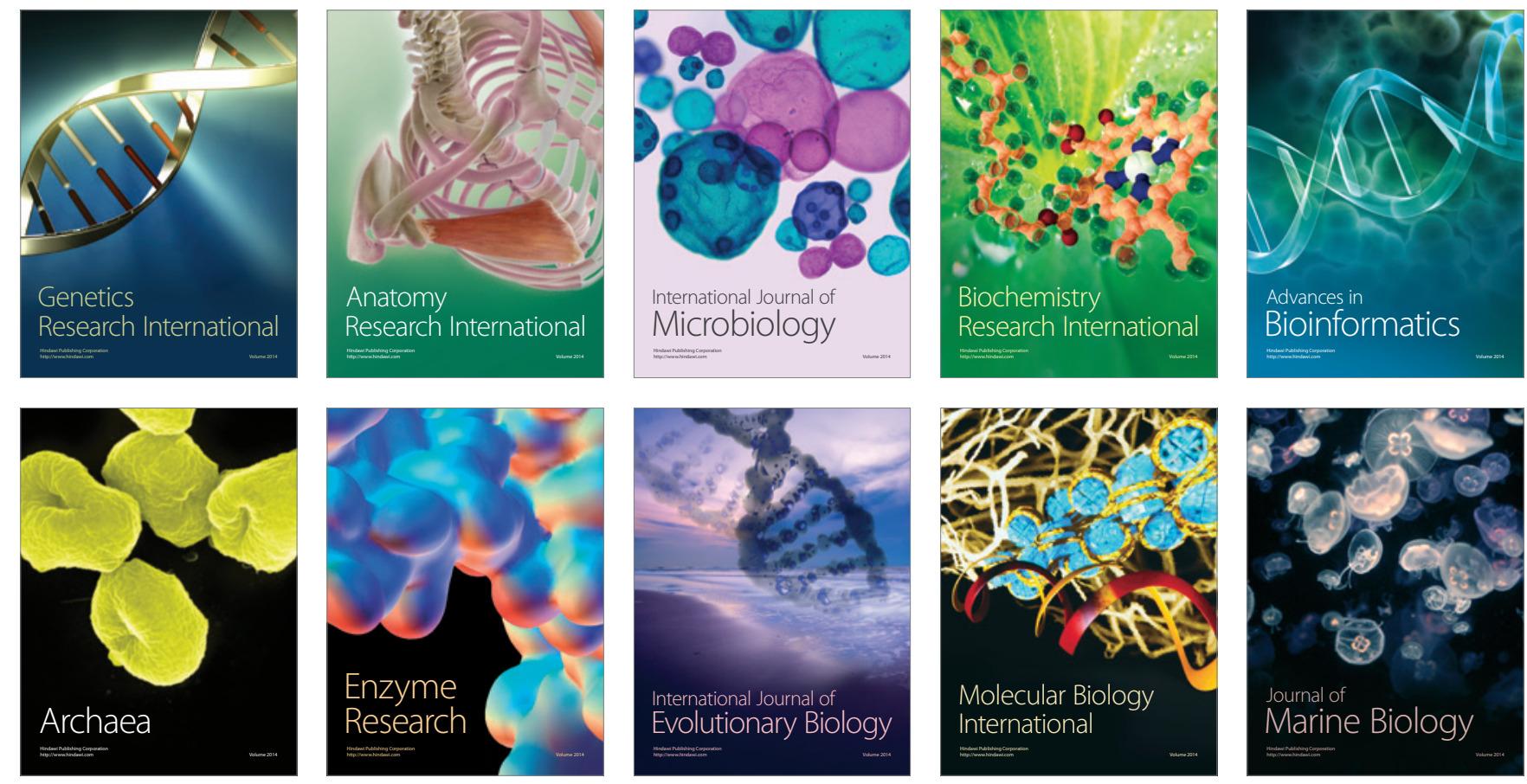